\title{
An integrative literature review on intimate partner violence against women in South Korea
}

\author{
Hye Young Min ${ }^{1}$, Jung Min Lee ${ }^{2}$, Yoonjung Kim \\ 'Division of Nursing Science, Graduate School, Ewha Womans University, Seoul, Korea \\ ${ }^{2}$ School of Nursing, University of North Carolina at Greensboro, USA
}

\begin{abstract}
Purpose: The purpose of this study was to analyze and synthesize the literature on intimate partner violence (IPV) against women in South Korea.

Methods: Whittemore and Knafl's integrative review method was used. Studies in English and Korean were searched in seven electronic databases using the following combination of terms: "Korea," "females or women or girls," "intimate partner violence or domestic violence or domestic abuse."

Results: Twenty-five studies were ultimately selected, all of which met the quality appraisal criteria with a grade of medium or higher, using Gough's weight of evidence. IPV was divided into marital violence and dating violence. Factors related to IPV were classified into intrapersonal, interpersonal, and social factors, and these three factors were linked together. Intrapersonal factors included general characteristics, perceptions, attitudes, psychological factors, and violent experiences. Interpersonal factors involved relationships with parents and partners. Finally, social factors and attributes were integrated into social support and influences on life.

Conclusion: In order to minimize and prevent harm to women from IPV when caring for women who experienced IPV, multiple factors should be considered. Specifically, general and psychological characteristics, perceptions and attitudes toward IPV, relationships with families and partners, and available social support systems and resources should be considered. Moreover, these findings will be helpful for assessing women or providing interventions for victims of violence. Finally, more diverse IPV studies should be conducted by nurses in the future.
\end{abstract}

Keywords: Domestic violence; Intimate partner violence; Review literature; Women
Received: June 23, 2020

Revised: November 1, 2020

Accepted: November 15, 2020

Corresponding author:

Yoonjung Kim

\# 104 Hellen Hall, 52 Ewhayeodaegil, Seodaemun-gu, Seoul 03760, Korea

Tel: +82-02-3277-2875

E-mail: kyjkyj1020@gmail.com

\section{Introduction}

Intimate partner violence (IPV), which includes controlling behavior, is one of the most common forms of violence against women [1]. In the study of García-Moreno et al. [2], IPV was defined as "any behavior within an intimate relationship that causes physical, psychological, or sexual harm to those in the relationship". In recent years, IPV has become an important public health issue. The incidence of violence against women, includ- ing sexual assault, is high worldwide [3], and despite ongoing movements and interest in fighting against violence - women, its incidence is not decreasing. According to a World Health Organization (WHO) report, 59\% of women aged between 15 and 49 years worldwide have experienced forced sexual or physical abuse by their partners at least once [3]. A 2018 report in Korea also reported that at least 824 women had been killed, and at least 602 women had been placed at risk of death, in the past 9 years by IPV [4]. This finding indicates that IPV is also a prob-

This is an Open Access article distributed under the terms of the Creative Commons Attribution License (http://creativecommons.org/licenses/by/4.0) which permits unrestricted use, distribution, and reproduction in any medium, provided the original work is properly cited.

Copyright (c) 2020 Korean Society of Women Health Nursing 


\section{Summary statement}

\section{- What is already known about this topic?}

Intimate partner violence (IPV) is often committed by dating and marital partners, and the incidence of sex crimes continues to increase.

\section{- What this paper adds}

IPV involves intrapersonal, interpersonal, and social factors, which are interlinked.

\section{- Implications for practice, education, and/or policy}

Nurses should be sufficiently prepared to prevent IPV and reduce the resulting harm. It is necessary to improve nurses' knowledge of related factors through education and to apply that knowledge in nursing care.

lem that cannot be overlooked in Korea.

Violence, caused by a power imbalance in close relationships, has recently been acknowledged as a serious social problem that needs to be addressed [5]. The power imbalance within traditional gender norms and close relationships directly or indirectly increases the vulnerability to violence within close relationships, causing the victim to be receptive to the other party's violent behavior [6]. Moreover, traditional gender norms and power disparities increase society's tendency to accept traditional gender roles and prevent women from flatly rejecting male proposals for sex. This increases the vulnerability of women to become sexual victims and exacerbates their inability to refuse unprotected sex $[6,7]$. Previous studies $[2,8]$ have shown that women with more stringent traditional gender norms have lower sexual self-assertion and self-efficacy within relationships, leading to an increase in the likelihood of experiencing sexual violence from their partners. These findings show that people who have no power in sexual relationships and who have a low level of sexual self-assertion are vulnerable to unwanted physical and mental harm, and are less capable of making actions or decisions related to sexual activities on their own. Women with these characteristics are more vulnerable to IPV, and efforts are needed to reduce its harm.

Sexual violence, in particular, is often caused by unilateral coercion during dating, without consent from the partner. IPV is often committed by dating partners [7], and the incidence of sex crimes (e.g., sexual violence, dating violence; wife-beating) continues to grow in Korea [9]. The percentage of people who reported dating violence reported in South Korea increased from $16.2 \%$ in 2016 to $19.9 \%$ in 2017 [10]. In addition, most perpetrators were male, and it was found that people in their $20 \mathrm{~s}$ experienced more dating violence than in other age groups [10]. Another Korean study [11] also reported similar results: about $97 \%$ of victims of sexual violence were women. Of these, $64.4 \%$ were adult women, and $55.7 \%$ were in their 20 s.

The physical or mental harm of violence against women is severe, both in the short and long term [12]. Victims of violence need immediate and long-term health care and psychological treatment. Violent experiences such as sexual assault, physical abuse, and stalking cause physical harm, which may include sexually transmitted diseases, as well as mental disorders such as post-traumatic disorder, depression, and insomnia [12]. In addition, because IPV occurs regularly and repeatedly due to the nature of the relationship between the victim and the perpetrator, women exposed to harm experience difficulties in forming supportive social relationships, have poor subjective health, and face limitations in daily activities $[3,13]$. Furthermore, violence reduces women's ability to work and opportunities to care for their families and contribute to society. Children who witness violence at home are also more likely to have behavioral and emotional problems [14].

There is a lack of systematic reviews on IPV experienced by Korean women, as well as a lack of research in Korea that distinguishes gender and power on IPV against women. However, since IPV has recently been recognized to be caused by power imbalances within close relationships, integrated consideration of existing research on IPV is essential. Therefore, this study sought to analyze and evaluate studies of IPV against Korean women, with the aim of identifying the characteristics and attributes of prior studies related to IPV against Korean women.

\section{Methods}

Ethics statement: This study is a literature review of previously published studies and was therefore exempt from institutional review board approval. 


\section{Research design}

This study is an integrative literature review that comprehensively analyzed research related to IPV against Korean women.

\section{Research procedure}

In accordance with the integrative review guidelines of Whittemore and Knafl [15], the research questions were clarified; the literature was searched and selected; data evaluation, data analysis, and semantic analysis of selected papers were conducted; and five stages of integrated data extraction were carried out. In addition, a quality assessment of the selected studies was conducted using Gough's weight of evidence (WOE) [16].

\section{Clarifying the research question}

To clarify the research problems and objectives, the researchers described the research question and objectives at the beginning of the study. The main research question of this study was, 'What does the nature of the studies related to IPV against Korean women show?'

\section{Search and selection of literature}

The researchers wanted to find meaningful and appropriate materials that fit the research problems and objectives, and recorded the process in detail to increase the accuracy of the literature search and the reliability of the research. Through meetings, the three researchers prepared the final analysis based on the following selection criteria. The criteria for selecting the papers included in the literature review were (1) studies of Korean women, (2) studies that included the keyword "intimate partner violence," (3) research papers published in Korean and international journals after peer review, and (4) studies in English or Korean. The criteria for exclusion from the literature review were (1) studies in which the study design was arbitration to see the effects of a literature review, tool development, and programs; (2) studies including males (because a separate analysis was not possible); and (3) government reports, dissertations, letters to editors, or papers published in academic conferences.

The first search and analysis of the literature was conducted from November 16 to December 16, 2019, and a second search was conducted from October 12 to October 21, 2020. There were no restrictions on the year of publication of the searched papers. The following three databases (DBs) were used: the Korean Studies Information Service System (KISS), the Research Information Sharing Service (RISS), and the National Digital Science Library (NDSL) in Korea and the keywords used for the search were a combination of "women," "IPV," "intimate partner violence," "close relationship violence," and "close part- ner violence." For international DBs, four search engines were used: Cumulative Index to Nursing and Allied Health Literature (CINAHL), PubMed, PsycINFO, and Scopus and the keywords were a combination of "Korea*," "females or women or girls," and "domestic violence or domestic abuse or intimate partner violence" (Supplementary Table 1).

Using the combined search terms, a total of 675 studies were identified, including 249 studies from Korean DBs and 426 studies from foreign DBs. From this pool, the titles and the abstracts were reviewed based on the selection criteria. In total, 334 studies and 291 duplicates were excluded. After carefully examining the full text of the remaining 50 studies and further applying exclusion criteria, we finally selected 25 studies [1741] (Figure 1).

\section{Evaluation of data}

To evaluate the quality of the selected literature, Gough's WOE [16] was used, and researchers assessed whether the research problems, research purposes, methods, selection of subjects, grounds, and results were described in the selected literature. The first item (WOE a) of the weighted value was evaluated with a focus on the context and evidence of the study by assessing the consistency and integration of the evidence presented in the study in relation to the research problem. The second item (WOE $b$ ) was to determine whether the form of evidence presented in the study in relation to the research question was consistent with the study question and purpose, thereby evaluating

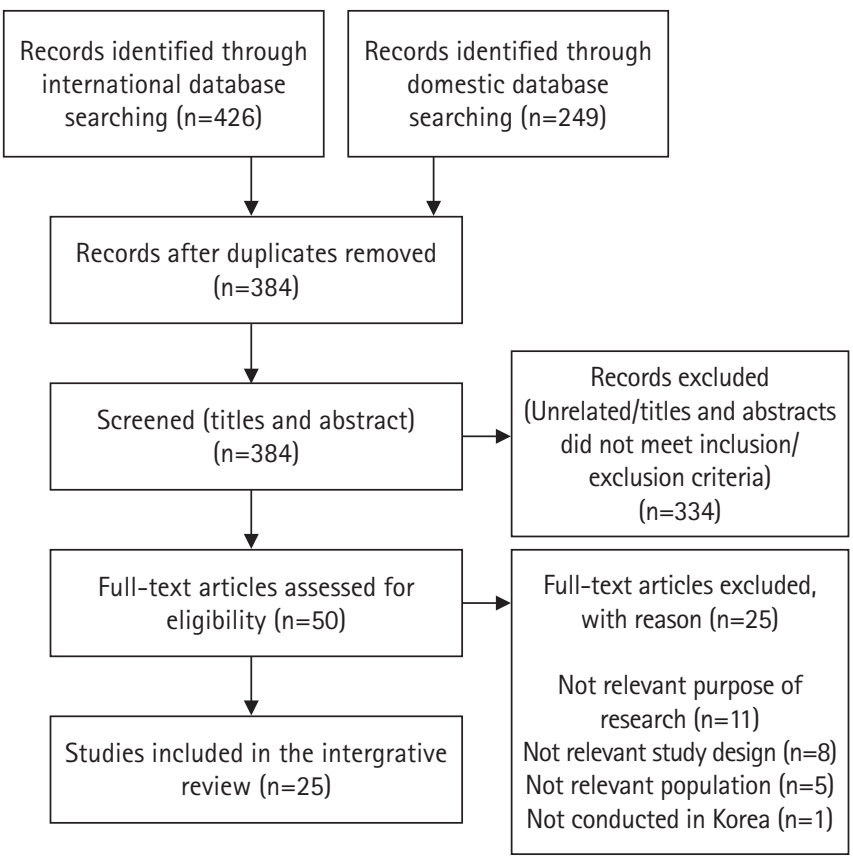

Figure 1. Flow diagram of study selection. 
whether the study was properly designed for the study question and purpose. The third item (WOE c) evaluated whether the research methods or research analyses were appropriate for the research problems, and the studies were evaluated with items such as selection of subjects, data collection and data analysis, and ethical aspects. Finally, the fourth item (WOE d) was evaluated comprehensively based on the three preceding items. A study was classified as "medium" or "high" if two or more of the three items evaluated earlier were "high" or "medium." However, in Gough's study [16], there was no clear standard for ratings based on the evaluation scores, so in this study, we referred to the study of Haßler et al. [42], which clearly presented evaluation criteria using Gough's WOE.

\section{Analyzing data and interpreting meaning}

Analyzing data and interpreting meaning are steps to analyze the finally selected research through quality evaluation and to synthesize its meaning. The researchers reviewed all the data independently, then organized and analyzed them individually. When the researchers compiled the selected papers, they extracted the year of publication, study design, participants, purpose of research and research questions, variables, and results of research, and also discussed what other selected studies found on IPV among Korean women. Subsequently, each of the summaries was compared and reviewed through regular meetings, and six offline and online meetings were conducted to coordinate opinions and to reach agreement on discrepancies in the data. In addition, the decision-making and progress processes from the beginning to the end of the study were described in detail so that other researchers could clearly understand these aspects of the study and reach a reasonable level of agreement with the opinions that emerged through the analysis.

\section{Integration of data}

The final step of the integrated consideration of study findings was to present how the attributes identified through the researchers' consensus were derived from the data. The analysis was conducted using the reference matrix to derive the properties of the research, and the final properties were confirmed by checking whether the derived properties were closely linked to the main data.

\section{Results}

Upon quality assessment, all 25 studies on IPV against Korean women analyzed in this study used data of medium or higher (Table 1). The characteristics of the analyzed studies were as follows (Table 2); fifteen studies $(60.0 \%)$ were conducted among married women and 10 studies (40.0\%) were conducted among single women. Quantitative studies were the most common (60.0\%), of which 12 (48.0\%) were descriptive studies. There were nine qualitative studies $(36.0 \%)$ and one mixedmethods study (4.0\%). Four studies on intimate relationship violence against women were published before 2011, 10 were published between 2011 and 2015, and 11 were published from 2016 to the present, indicating that studies related to women's violence have been steadily published. The main variables of the reviewed papers related to marital violence (32.0\%), dating violence $(40.0 \%)$, and victims' beliefs, attitudes, and influencing factors for each type of abuse (28.0\%). Most studies were conducted in fields in the social sciences such as social welfare $(n=7)$, psychology $(n=5)$, and law and criminology $(n=5)$, followed by nursing $(n=5)$ and medicine $(n=1)$. Although there were relatively few studies in the health field, the four studies published within the last 2 years were from nursing, suggesting an increasing interest in the study of IPV in nursing research.

Table 1. Quality appraisal of reviewed articles ( $\mathrm{N}=25)$

\begin{tabular}{|c|c|c|}
\hline First author [Ref No.] & Year & Gough's WOE (WOE a, WOE b, WOE c, WOE d) \\
\hline Ko [35] & 2020 & $\mathrm{H}, \mathrm{H}, \mathrm{H}, \mathrm{H}$ \\
\hline Park [34] & 2020 & $\mathrm{H}, \mathrm{H}, \mathrm{H}, \mathrm{H}$ \\
\hline Kwon [29] & 2019 & $\mathrm{H}, \mathrm{H}, \mathrm{H}, \mathrm{H}$ \\
\hline Kwak [23] & 2018 & $\mathrm{H}, \mathrm{H}, \mathrm{M}, \mathrm{H}$ \\
\hline You [31] & 2018 & $\mathrm{H}, \mathrm{H}, \mathrm{H}, \mathrm{H}$ \\
\hline Hong [20] & 2017 & $H, H, M, H$ \\
\hline Kim [19] & 2017 & $\mathrm{H}, \mathrm{H}, \mathrm{H}, \mathrm{H}$ \\
\hline Woo [32] & 2017 & $\mathrm{H}_{1} \mathrm{H}_{1} \mathrm{H}, \mathrm{H}$ \\
\hline Park [41] & 2017 & $H, H, H, H$ \\
\hline Shon [39] & 2016 & $H, H, M, H$ \\
\hline Park [24] & 2016 & $H, H, M, H$ \\
\hline Hong [26] & 2015 & $H, H, M, H$ \\
\hline Bae [33] & 2014 & $\mathrm{H}, \mathrm{H}, \mathrm{H}, \mathrm{H}$ \\
\hline Choi [17] & 2014 & $H, H, M, H$ \\
\hline Park [30] & 2014 & $H, H, M, H$ \\
\hline Jennings [21] & 2014 & $\mathrm{H}, \mathrm{H}, \mathrm{H}, \mathrm{H}$ \\
\hline Kim [38] & 2013 & $\mathrm{H}, \mathrm{H}, \mathrm{M}, \mathrm{H}$ \\
\hline Kim [37] & 2013 & $\mathrm{H}, \mathrm{H}, \mathrm{M}, \mathrm{H}$ \\
\hline Han [36] & 2012 & $\mathrm{H}, \mathrm{H}, \mathrm{M}, \mathrm{H}$ \\
\hline Park [25] & 2012 & $H, H, M, H$ \\
\hline Hong [40] & 2011 & $\mathrm{H}, \mathrm{H}, \mathrm{M}, \mathrm{H}$ \\
\hline Kim [27] & 2010 & $\mathrm{H}, \mathrm{H}, \mathrm{H}, \mathrm{H}$ \\
\hline Kim [28] & 2010 & $H, H, M, H$ \\
\hline Kim [18] & 2009 & $\mathrm{H}, \mathrm{H}, \mathrm{M}, \mathrm{H}$ \\
\hline Kim [22] & 2009 & $\mathrm{H}, \mathrm{H}, \mathrm{M}, \mathrm{H}$ \\
\hline
\end{tabular}

Ref No:: reference number; WOE: weight of evidence.

$\mathrm{H}$ : high; M: medium; WOE a: methodological trustworthiness; WOE b: methodological relevance; WOE c: topic relevance; WOE d: overall score. 
Table 2. General characteristics of the included studies $(\mathrm{N}=25)$

\begin{tabular}{|c|c|c|c|}
\hline Characteristic & Categories & n $(\%)$ & Reference number \\
\hline \multirow[t]{2}{*}{ Participants } & Single women & $15(60.0)$ & [17], [19], [20], [21], [23], [25], [27], [29], [31], [32], [34-36], [39], [41] \\
\hline & Married women & $10(40.0)$ & [18], [22], [24], [26], [28], [30], [32], [33], [38], [40] \\
\hline \multirow[t]{4}{*}{ Research design } & Quantitative descriptive study & $12(48.0)$ & {$[17-28]$} \\
\hline & Qualitative study & $9(36.0)$ & [29-35], [40], [41] \\
\hline & Secondary data analysis & $3(12.0)$ & [36-38] \\
\hline & Mixed-methods study & $1(4.0)$ & [39] \\
\hline \multirow[t]{3}{*}{ Publication year } & Before 2011 & $4(16.0)$ & [18], [22], [27], [28] \\
\hline & 2011-2015 & $10(40.0)$ & [17], [21], [25], [26], [30], [33], [37], [38], [40] \\
\hline & 2016 and beyond & $11(44.0)$ & [19], [20], [23], [24], [29], [31], [32], [34], [35], [39], [41] \\
\hline \multirow[t]{4}{*}{ Sample size } & Less than 100 & $13(52.0)$ & [17], [29], [20], [28-35], [40], [41] \\
\hline & $101-500$ & $7(28.0)$ & [23-27], [36], [37] \\
\hline & $501-1,000$ & $2(8.0)$ & [22], [39] \\
\hline & $>1,000$ & $3(12.0)$ & [18], [21], [38] \\
\hline \multirow[t]{3}{*}{ Main variables } & Marital violence & $8(32.0)$ & [18], [20], [24], [26], [28], [30], [33], [40] \\
\hline & Dating violence & $10(40.0)$ & [17], [21], [22], [29], [31], [32], [34], [35], [39], [41] \\
\hline & Factors affecting violence & 7 (28.0) & [19], [23], [25], [27], [36], [37], [38] \\
\hline \multirow[t]{6}{*}{ Study field } & Social welfare & $7(28.0)$ & [18], [19], [24], [26], [30], [32], [38] \\
\hline & Counseling & $2(8.0)$ & [31], [41] \\
\hline & Psychology & $5(20.0)$ & {$[17],[20],[25],[32],[40]$} \\
\hline & Law and criminology & $5(20.0)$ & [21], [22], [26], [28], [37] \\
\hline & Nursing & $5(20.0)$ & [23], [29], [34-36] \\
\hline & Medicine & $1(4.0)$ & [39] \\
\hline
\end{tabular}

\section{Analysis of data for selected papers}

In this study, 25 studies on IPV were analyzed according to the study questions (Table 3). The 12 descriptive studies presented research on experiences of IPV [17-19], influencing factors [20$25]$, the legal and support system [26,27], and women who relied on the perpetrators of IPV [28]. The nine qualitative studies explored experiences of IPV [29-32], women's experience of building their identity after IPV [33], experiences of seeking help to overcome IPV [34], and experiences after IPV ended $[17,35]$. Quantitative studies investigated the level of harm caused by IPV [36], predictors of risk [37], the relationship of IPV with depression [38], and gender awareness and self-assertion [39]. Among the major variables used in IPV studies were demographic and sociological characteristics related to marital status and household income $[22,28,38]$, stereotypes such as dating violence beliefs or tolerance $[17,24,25,36,39]$, and sexual self-assertion related to perceptions of violence against women $[23,25,39]$. In addition, studies on coping $[29,31,32,34]$ and the coping process $[31,32,35]$ were conducted in studies dealing with post-IPV experiences.

Women's experiences of spousal violence were closely related to their experiences of parental verbal violence in childhood [18] and subsequently were associated with women's depres- sion, stress, and diminished self-esteem. The most commonly investigated risk factor was the experience of parental violence against the child, and if violence was experienced as a child, this was likely to become transposed to a violent relationship in adulthood $[33,38]$. Conversely, a study that evaluated the causal relationship between child abuse victims and adult dating violence offenders [21] found that experiencing child abuse by parents did not have a statistically significant impact on subsequent physical dating violence and youth harm. Those who experienced parental violence or watched their mothers be abused since childhood $[30,33]$ or grew up in patriarchal home environments $[22,28]$ developed stereotypes about gender roles and showed low sexual self-assertion as women $[17,37]$. Experiences of parental violence before adulthood affect interpersonal relationships due to depression and stress, and increase the risk of intimate violence after adulthood. The results of analyzing the selected studies suggest that steps must be taken to educate parents on how to correct their children's behavior without physical punishment or verbal violence, and that mediation programs to prevent marital violence in the community should be implemented more actively.

Some of the studies $[24,25,29,32,41]$ regarding dating violence showed that gender stereotypes and sexual self-assertion 


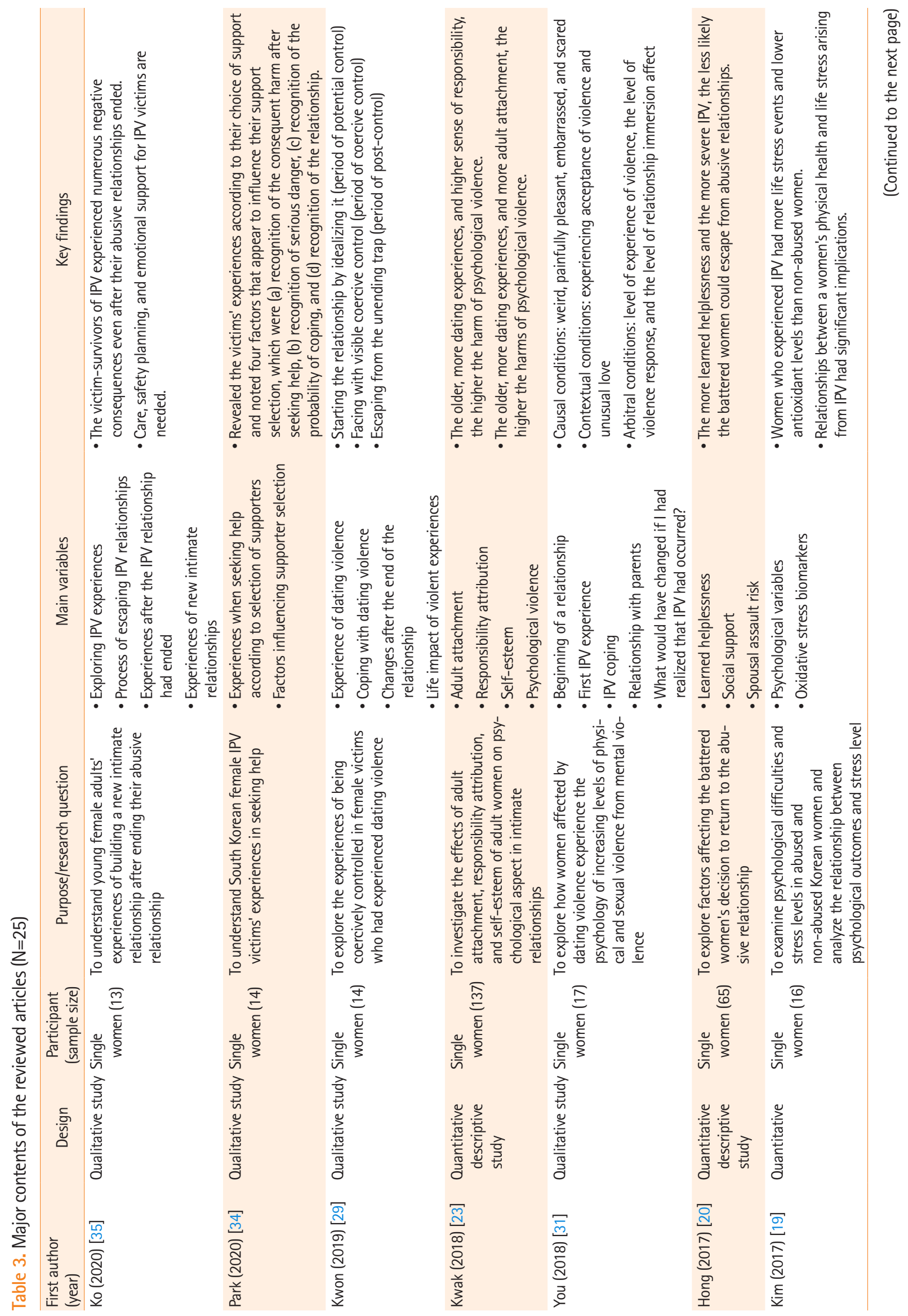




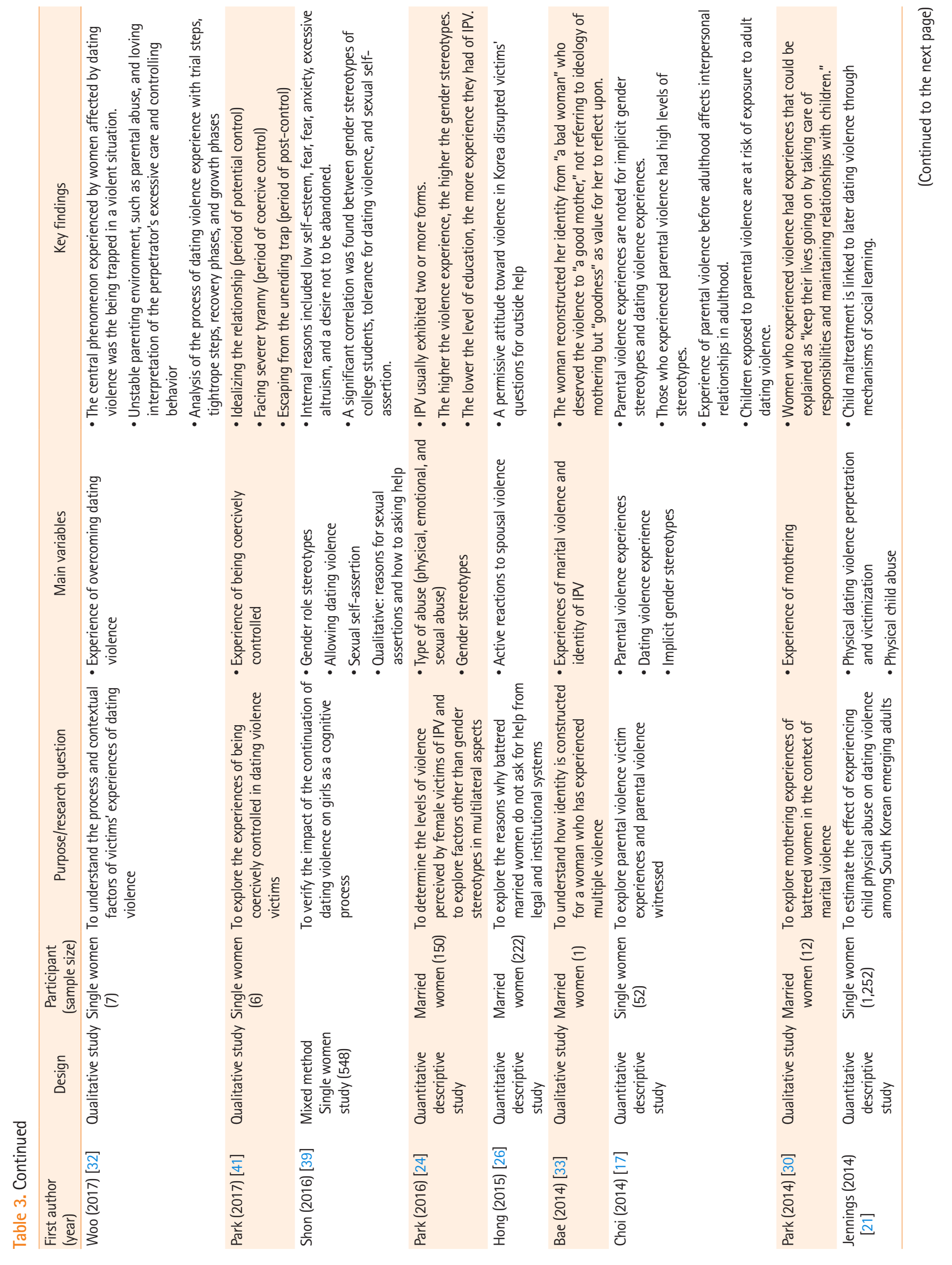




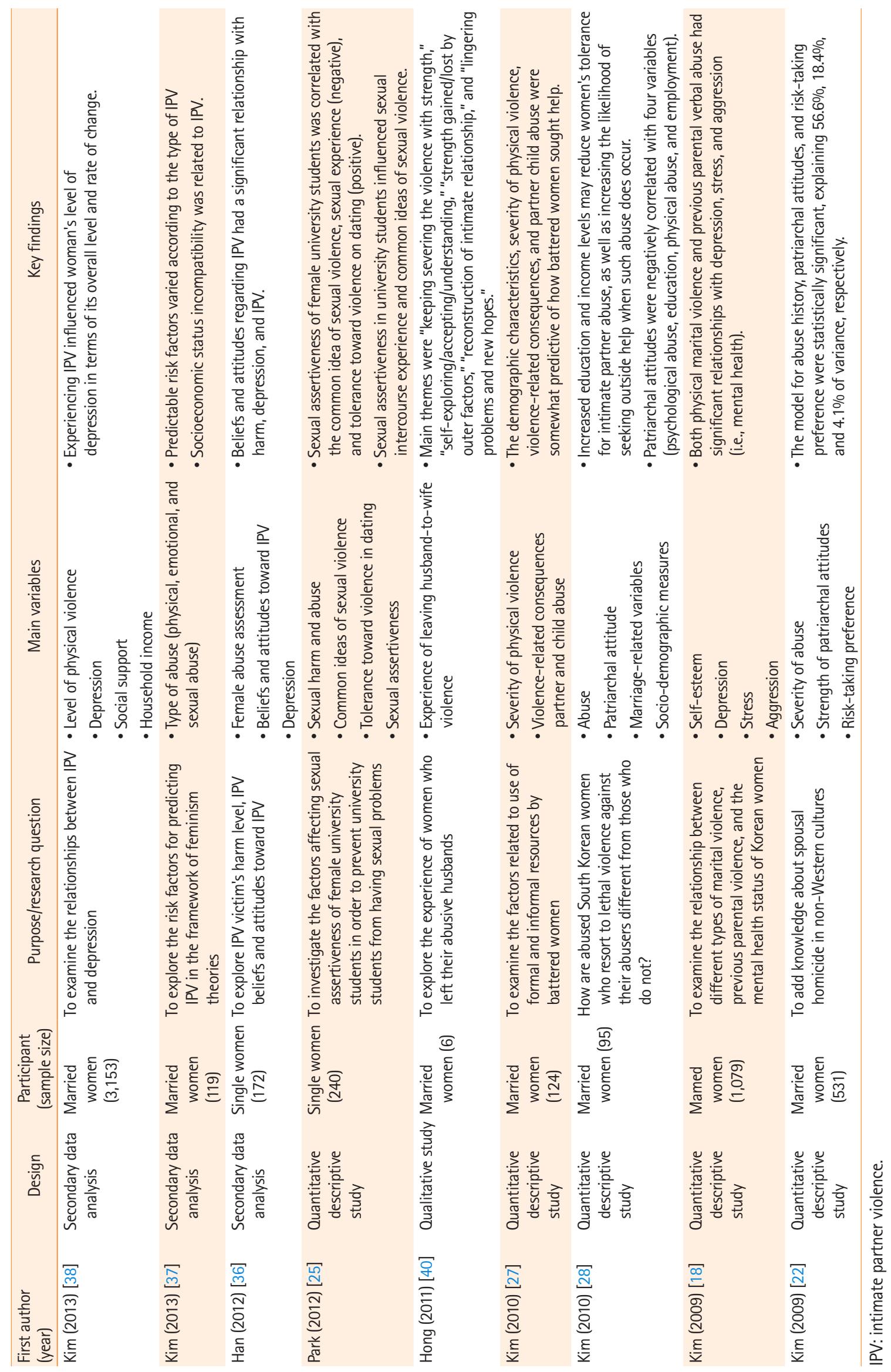


affected the acceptance of violence in close relationships $[24,25]$. Among victimized women exposed to parental violence in childhood, a learned sense of helplessness about violence appeared [20], the severity of abuse was not recognized in close relationships [28], and tolerance for violence was confirmed to be different $[25,39]$. In addition, women who experienced parental violence due to exposure to patriarchal attitudes in childhood were found to be at risk of becoming perpetrators and victims of violence in the future $[17,22,24,28,31]$. Perceptions of violence in intimate relationships varied among women who were victims depending on their knowledge about sexual violence [25] and implicit gender stereotyping [17,24,39], which has been shown to affect woman's beliefs in and attitudes toward violence [36]. Adult attachment, self-understanding, self-impact factors [18,23], and sexual self-assertion [25,39] were found to affect post-violence attitudes in close relationships.

A secondary study of IPV $[27,36]$ assessed the level of harm to victimized women and their beliefs, attitudes, and depression about violence. Tolerances or attitudes toward violence against women in Korea were related to educational level [22,24,27,38], previous experiences of parental violence $[17,19,21]$, and adult attachment $[23,30,33]$. An analysis of the general characteristics of women who had experienced violence $[23,24,27]$ showed that the awareness, severity and tolerance of violence were associated with unstable employment status, low income level, and low education level. Among women affected by IPV, with high levels of education and easy access to social support services, attempts have been made to overcome experiences of violence by seeking help and using support systems in the local community $[20,25,26]$. In addition, studies conducted among women revealed that higher levels of sexual assertiveness were associated with less tolerance of dating violence and lower gender role stereotypes $[25,39]$.

In this review, studies on the coping and overcoming of IPV among Korean women were examined, including changes after the relationship with the perpetrator ends $[29,35]$, and requests for help in the process of overcoming IPV [34]. Besides, the results of this review, IPV should be assessed in the local community as a way to overcome violence $[18,21,28]$, and in order to prevent violence, education on gender stereotypes $[22,24,26]$ and violence $[17-19,28,39]$ is needed. It was also found that women who already have experienced IPV need education for increased sensitivity to violence, as well as counseling and psychotherapy to prevent subsequent trauma, such as depression and distress $[20,29,36,38,41]$.

\section{Interpretation of meaning}

The factors that influenced women before the occurrence of IPV included previous experiences of violence, growth environment, beliefs and attitudes, gender stereotypes, gender stereotypes, and sexual self-assertion. The types of IPV were broadly divided into marital violence and dating violence, which in turn could be classified as physical, mental, and sexual violence. However, types of violence in close relationships occurred in association with each other rather than independently. After the occurrence of IPV, the impacts of IPV on women included depression, stress, and anxiety. The victim's attitude toward overcoming violence and ways of coping with it affect the likelihood of becoming a victim of IPV again.

\section{Integration of data}

In this study, IPV-related factors were grouped into intrapersonal factors, interpersonal factors, and social factors, and these three factors were linked together (Table 4). First, intrapersonal factors included women's general characteristics and psychological factors, awareness and attitudes toward IPV, and past experiences of IPV. Specifically, psychological factors included depression, stress, self-understanding, awareness of IPV, knowledge and beliefs, sexual self-assertion, and gender role stereotypes, including awareness of IPV and attitudes toward IPV. In addition, past experiences of IPV included parental violence and child abuse. In addition, the findings showed that personal experiences on parental violence or dating violence and gender norms influenced individuals' ability to ask for help or manage violence in close relationships. Second, interpersonal factors referred to the relationships between individuals, in addition to the characteristics of the individual. These relationships can be divided into parents and partners, including spouses. Women who suffered violence in intimate relationships were affected by witnessing patriarchal attitudes, parental violence, and mother-targeted violence in childhood. Their relationships with partners affected their experiences of marital violence, the severity of physical violence, the risk of spousal assault, the consequences of violence, their active response to spousal violence, and their tolerance of dating violence. The researchers identified the formation of intimate violence in relationships with new partners and the risk that past victims may become perpetrators. Third, social factors included the impact of social support, such as the degree of counseling assistance, the relationship with the counselor, and the social support system after IPV. Furthermore, analyses of the influence of IPV on life before or after the harm showed that the victim-survivors of IPV experience numerous negative consequences even after their abusive relationships end $[32,35]$. 
Table 4. Integrative description of intimate partner violence

\begin{tabular}{lll}
\hline Attribute & Categories & Reference number \\
\hline Intrapersonal factor & General characteristics & [28], [30], [41] \\
& Perceptions of IPV & [19], [20], [25], [31], [36-38], [41] \\
& Attitudes toward IPV & [19], [22], [29], [31] \\
& Psychological factors & [18], [28], [37], [38] \\
Interpersonal factor & Violence experience & {$[19-21],[29-32],[34-36],[40]$} \\
Social factor & Relationships with parents & [23], [24], [30], [31], [41] \\
& Relationships with partner & [17], [24], [26-30], [33-35] \\
\hline
\end{tabular}

IPV: Intimate partner violence.

\section{Discussion}

This study found that research on IPV has been consistently published since 2010. The incidence of IPV in Korea has decreased compared to the past, but it still has a higher profile than that of other countries [43]. This warrants continuing attention. Most studies on IPV were conducted in the social sciences, and only five of the 25 studies were in nursing, suggesting the need for more intensive nursing research on IPV. The WHO recognizes IPV as a public health issue and emphasizes the need for an appropriate understanding among health care providers, including nurses [12]. Nurses are more likely to encounter IPV victims [44] and spend the longest time with patients compared to other health care providers [45]. Therefore, nurses must be sufficiently prepared to face and treat women who report instances of IPV. In a study of nurses' roles and preparation for IPV that was conducted in India [46], the results showed positive correlations among the level of educational preparedness, self-efficacy, and attitudes toward caring for women affected by IPV. Another study confirmed that simulation education for IPV was significantly helpful for preparing nursing students to care for women affected by IPV [47]. Thus, conducting more diverse studies of IPV for nursing education in Korea will be beneficial.

The factors related to IPV can be classified into intrapersonal, interpersonal, and social factors. Intrapersonal factors (e.g., depression, stress, and experiences of violence in the past) were identified as risk factors of IPV in a prior study [48], and therefore the psychological and social aspects of IPV must be assessed when caring for victims. In addition, for adult women who have not experienced IPV or have not suffered harm, psychological and social aspects should be assessed to prevent and minimize harm, and nurses must identify and link resources that can be utilized within the community in advance if necessary. As experiences of parental violence and child abuse while grow- ing up appears to affect adulthood, education about and screening for pre-adult IPV is also necessary [48]. So far, however, preventive education for IPV has been insufficient. Since violence prevention education mainly deals with sexual violence for school-age children, adolescents, and young adults [49,50], education should therefore include more a comprehensive and wider range of knowledge about IPV.

In addition, nurses should be prepared to consider combinations of intrapersonal factors. In other words, for nurses to deal with IPV, a wide range of understanding and knowledge is needed through education. If nurses are prepared to provide nursing care on the basis of a sufficient knowledge of IPV, they will have a positive attitude and self-efficacy when caring for women [46]. Therefore, if nurses understand the characteristics of women vulnerable to violence in intimate relationships (e.g., low education, being young and married) [43], and reflect that understanding in their care, they will be able to have a positive impact on the women as well as on themselves.

Second, interpersonal factors are those related to relationships with others in addition to individual characteristics. As the notion of IPV [1] involves all forms of violence in close relationships, IPV can be broadly divided into relationships with parents and partners, including previous parental violence, as well as dating violence. Therefore, in order to provide an assessment and mediation of IPV, it is necessary to implement parental education [18] as well as marital and partner education as precautionary interventions. Partner training should be aimed toward teenagers and college students who are potential partners in the future. The training should also include both male and female teenagers and college students, rather than targeting only men, considering the diverse gender identities of women.

Lastly, for social factors, women affected by IPV are more likely to comply with the perpetrator's demands than to break away from the violent relationship [29]. Thus, there is a need for interventions addressing social factors that enable women to 
overcome subsequent trauma. For example, attitudes toward IPV may be changed through counseling, which may help women to cope more effectively with the effects of IPV. For this purpose, at the community level, women exposed to danger should be assessed and protected from harm $[18,28]$. In addition, social and policy interventions are necessary. In Korea, the Framework Act on the Prevention of Violence against Women was enacted in 2018 to protect women from various forms of violence, including violence in close relationships. The Act was established following a nationwide descriptive survey, aimed to protect women who have been exposed to violence and to protect and support the victims. In addition, there are currently hotlines in Korea dedicated to women that provide counseling on violence against women, linkage and supportive resources for victims, training in preventing violence, and activities to increase awareness of IPV through telephone support [4]. Therefore, nurses need to be familiar with the laws enacted and related available resources to be able to make full use of social and policy aspects, as well as personal preparation.

This study has some limitations. The search was limited to research papers on IPV affecting women, in order to comprehensively examine the experience of IPV against Korean women. In addition, although the researchers made efforts to conduct a systematic and comprehensive search and selection, it is possible that some relevant papers were missed in the search phase or in the title and abstract review phase. Finally, as this study focused on research related to IPV against Korean women in Korea, there is a limit to generalizing the results to IPV against migrant women living in Korea or Korean women living abroad.

This study examined 25 studies on IPV against women in Korea and, through the meaning analysis of IPV, identified important factors that influenced IPV before its occurrence, types of violence upon occurrence, and the process of overcoming IPV after its occurrence. It was also confirmed that IPV affects women in terms of intrapersonal, interpersonal, and social factors, which are closely interlinked. The results of this study are significant in that they include matters that nurses may consider in preventing IPV and caring for victimized women. Specifically, general and psychological characteristics, perceptions and attitudes toward IPV, relationships with families and partners, and available social support systems and resources should be considered. The results of this study can be used for developing educational content in the required curriculum for teenagers and college students, by including content on intrapersonal, interpersonal, and social factors of IPV. The findings can also guide IPV assessment and the provision of arbitration for victims.

Since nursing research on IPV is limited, albeit having in- creased in the past 2 years, more IPV research by nurses is needed. This includes studies that focus on nurses, such as descriptive studies on nurses' knowledge and perceptions of IPV, as well as nursing education for IPV care readiness.

\section{ORCID}

Hye Young Min, https://orcid.org/0000-0002-0793-8718

Jung Min Lee, https://orcid.org/0000-0002-4916-5485

Yoonjung Kim, https://orcid.org/0000-0003-0104-3865

\section{Authors' contributions}

Conceptualization: Lee JM; Formal analysis: Min HY, Kim Y; Writing-original draft: all authors; Writing-review \& editing: all authors.

\section{Conflict of interest}

The authors declared no conflict of interest.

\section{Funding}

None.

\section{Data availability}

Please contact the corresponding author for data availability.

\section{Acknowledgments}

None.

\section{Supplementary materials}

Further details on supplementary materials are presented online (available at https://doi.org/10.4069/kjwhn.2020.11.15).

\section{References}

1. World Health Organization. Intimate partner violence 2012 [Internet]. Geneva: Author; 2013 [cited 2020 May 4]. Available from: https://apps.who.int/iris/bitstream/handle/10665/77432/WHO_RHR_12.36_eng.pdf;jsession$\mathrm{id}=$ 537B7B2F537EF0467E220D09B4056357? sequence $=1$

2. García-Moreno C, Hegarty K, d’Oliveira AF, Koziol-McLain J, Colombini M, Feder G. The health-systems response to vio- 
lence against women. Lancet. 2015;385(9977):1567-1579. https://doi.org/10.1016/S0140-6736(14)61837-7

3. World Health Organization. Global and regional estimates of violence against women: prevalence and health effects of intimate partner violence and non-partner sexual violence [Internet]. Geneva: Author; 2020 [cited 2020 May 4]. Available from: https://apps.who.int/iris/bitstream/handle/10665/ 85239/9789241564625_eng.pdf;jsessionid $=752435566 \mathrm{~F}$ 46328E16DE21C2016EB8? sequence $=1$

4. Task Force for the Framework Act on Gender-based Violence against Women. What is the need for gender-based violence against women's violence? [Internet]. Seoul: Womenlink; 2018 [cited 2020 May 4]. Available from: https://www.womenlink.or.kr/minwoo_actions/20828

5. Hong YO, Yeon SJ, Ju SH. Violence against women: focused on intimate partner violence. Seoul: Korean Institute of Criminology; 2015.

6. Park J, Shin N, Park H. A convergence study on the influence of media violence acceptance and violence perception on dating violence in university students. J Korea Converg Soc. 2019;10(4):237-246. https://doi.org/10.15207/JKCS.2019. 10.4.237.

7. Lee ES, Kang HS. Factors influencing the experiences of sexual violence victims while dating as college students. Korean J Women Health Nurs. 2010;16(3):266-275. https://doi. org/10.4069/kjwhn.2010.16.3.266

8. Korea Women's Hotline. The gauge of anger 2017: statistical analysis of women killed by intimate men [Internet]. Seoul: Author; 2018 [cited 2020 Nov 8]. Available from: http://hotline.or.kr/board_statistics/37289? ckattempt = 1

9. Ministry of Government Legislation. Violence prevention against women act [Internet]. Seoul: Author; 2018 [cited 2020 Apr 3]. Available from: http://www.law.go.kr/LSW/ nwRvsLsInfoR.do?lsiSeq $=206085$

10. Statistics Korea. Women's life as a whole in 2018 statistics [Internet]. Daejeon: Author; 2018 [cited 2020 Nov 8]. Available from: http://kostat.go.kr/portal/korea/kor_nw/1/6/1/index. board bmode $=$ read \&aSeq $=368636 \&$ pageNo $=1 \&$ rowNum $=10 \&$ amSeq $=\&$ sTarget $=\& s$ Txt $=$

11. Shim CS, Lee YS, Oh SH. Study on the relationship between gender-role stereotypes, sexual assault awareness and permissible limit in the college students. Korean J Women Health Nurs. 2012;18(2):117-125. https://doi.org/10.4069\%2Fkjwhn.2012.18.2.117

12. Department of Reproductive Health and Research, World Health Organization; London School of Hygiene and Tropical Medicine; South African Medical Research Council.
Global and regional estimates of violence against women [Internet]. Geneva: Author; 2013 [cited 2020 Nov 8]. Available from: https://www.who.int/publications/i/item/9789241 564625.

13. World Health Organization. Multi-country study on women's health and domestic violence against women: report - initial results on prevalence, health outcomes and women's responses [Internet]. Geneva: Author; 2005 [cited 2020 Nov 8]. Available from: https:// www.who.int/reproductivehealth/ publications/violence/24159358X/en/

14. United Nations Women Headquarters. Ending violence against women: from words to action. Study of the secretary general [Internet]. New York: Author; 2006 [cited 2020 Nov 8]. Available from https://www.unwomen.org/en/digital-library/publications/2006/1/ending-violence-against-womenfrom-words-to-action-study-of-the-secretary-general

15. Whittemore R, Knafl K. The integrative review: updated methodology. J Adv Nurs. 2005;52(5):546-553. https://doi. org/10.1111/j.1365-2648.2005.03621.x

16. Gough D. Weight of Evidence: a framework for the appraisal of the quality and relevance of evidence. Res Pap Educ. 2007;22(2):213-228. https://doi.org/10.1080/02671520701296189

17. Choi Y, Song W. The effects of college women's domestic violence experiences on dating violence exposure: mediated effect of explicit and implicit gender stereotypes. Korean J Woman Psychol. 2014;19(4):433-446. https://doi.org/10.18205/ kpa.2014.19.4.005

18. Kim J, Park S, Emery CR. The incidence and impact of family violence on mental health among South Korean women: results of a national survey. J Fam Viol. 2009;24:193-202. https://doi.org/10.1007/s10896-008-9220-5

19. Kim JY, Lee JH, Song HJ, Kim DG, Yim YS. Relationships between psychosocial difficulties and oxidative stress biomarkers in women subject to intimate partner violence. Health Soc Work. 2017;42(1):41-47. https://doi.org/10.1093/hsw/ hlw053

20. Hong JH, Kwon HJ, Yoon SI, Lee SJ. Factors to affect battered women's decisions going back to the abused relationship: focused on the learned helplessness, social support, and the risk of domestic violence. Korean J Woman Psychol. 2017;22(1):122. https://doi.org/10.18205/kpa.2017.22.1.001

21. Jennings WG, Park M, Richards TN, Tomsich E, Gover A, Powers RA. Exploring the relationship between child physical abuse and adult dating violence using a causal inference approach in an emerging adult population in South Korea. Child Abuse Negl. 2014;38(12):1902-1913. https://doi.org/ 10.1016/j.chiabu.2014.08.014 
22. Kim B, Titterington VB. Abused South Korean women: a comparison of those who do and those who do not resort to lethal violence. Int J Offender Ther Comp Criminol. 2009; 53(1):93-112. https://doi.org/10.1177/0306624X07312772

23. Kwak SJ, Woo CH, Kim NY. Effect of adult attachment, attribution of responsibility, and self-esteem on psychological violence from intimate partner in women. J Korea Converg Soc. 2018;9(1):75-84. https://doi.org/10.15207/JKCS.2018. 9.1.075

24. Park KY, Kim IG. Analysis of violence perceived by female victims of domestic violence: gender stereotype as mediation effect. J Soc Welf Manag. 2016;3(2):19-39.

25. Park SM, Song W. The effects of rape myth and tolerance towards violence on dating to the sexual assertiveness in college women. Korean J Women Psychol. 2012;17(2):265-280. https://doi.org/10.18205/kpa.2012.17.2.010

26. Hong TK. An analysis of effective factors to intimate partner violence victims' active response: focusing on ecological theory. J Police Sci. 2015;15(3):147-174.

27. Kim JY, Lee JH, Han S, Song HJ. Factors influencing hazardous drinking of women who experienced intimate partner violence. Korean J Soc Welf. 2010;64(2):275-296. https:/doi. org/10.20970/kasw.2010.62.4.012

28. Kim B, Titterington VB, Kim Y, Wells W. Domestic violence and South Korean women: the cultural context and alternative experiences. Violence Vict. 2010;25(6):814-830. https:// doi.org/10.1891/0886-6708.25.6.814

29. Kwon J, Park S. Experiencing coercive control in female victims of dating violence. J Korean Acad Nurs. 2019;49(1):4658. https://doi.org/10.4040/jkan.2019.49.1.46.

30. Park EJ. Construction of mothering in the context of domestic violence and battered women's agency. J Korean Women's Stud. 2014;30(1):173-212.

31. You Y, Park K. A qualitative study on the counseling experience of female victims of dating violence. The Korean J Counsel Psychother. 2018;30(4):955-983.

32. Woo HJ, Jang SH, Kwon H. A study on the overcoming experience of women suffering from dating violence. Korean J Woman Psychol. 2017;22(3):315-335. https://doi.org/10. 18205/kpa.2017.22.3.001

33. Bae YM. The experienced violence and identity of domestic violence victim: narrative inquiry. J Fam Relat. 2014;8(4):333.

34. Park S, Ko Y. Victims of intimate partner violence in South Korea: experiences in seeking help based on support selection. Violence Against Women. 2020 Feb 24 [Epub]. https:// doi.org/10.1177/1077801220905638
35. Ko Y, Park S. Building a new intimate relationship after experiencing intimate partner violence in victim-survivors of South Korea. J Interpers Violence. 2020;35(1-2):3-34. https:// doi.org/10.1177/0886260518814265

36. Han YR. Beliefs and attitudes toward intimate partner violence and depression in victims of intimate partner violence dwelling in the community. J Korean Public Health Nurs. 2012;26(3):532-546. https://doi.org/10.5932/JKPHN.2012. 26.3.532

37. Kim EY, Yun M, Park S. An examination on the risk factors predicting female intimate partner violence victims' physical, emotional and sexual abuse. Korean Police Stud Rev. 2013; 12(2):49-78.

38. Kim J, Lee J. Prospective study on the reciprocal relationship between intimate partner violence and depression among women in Korea. Soc Sci Med. 2013;99:42-48. https://doi. org/10.1016/j.socscimed.2013.10.014

39. Shon KS, Jeong SM. The mediation effects of sexual assertiveness between gender-role stereotypes and dating violence acceptability of female college students. Korean J Woman Psychol. 2016;21(3):441-457.

40. Hong SH, Ahn HN. A qualitative study change processes in women who left their abusive husbands. Korean J Woman Psychol. 2011;16(1):69-96.

41. Park K, You Y. A study on the psychological innerness in female victims of dating violence. Korean J Counsel Psychother. 2017;29(3):711-742. https://doi.org/10.23844/kjcp. 2017.08.29.3.711

42. Haßler B, Major L, Hennessy S. Tablet use in schools: a critical review of the evidence for learning outcomes. J Comput Assist Learn. 2016;32(2):139-156. https://doi.org/10.1111/ jcal.12123.

43. Kim JY, Oh S, Nam SI. Prevalence and trends in domestic violence in South Korea: findings from national surveys. J Interpers Violence. 2016;31(8):1554-1576. https://doi.org/10. $1177 / 0886260514567960$.

44. Campbell JC. Health consequences of intimate partner violence. Lancet. 2002;359(9314):1331-1336. https://doi.org/ 10.1016/S0140-6736(02)08336-8.

45. Kufe DW, Pollock RE, Weichselbaum RR, Bast RC Jr, Gansler TS, Holland JF, et al., editors. Holland-Frei cancer medicine. 6th ed. Hamilton (ON): BC Decker; 2003.

46. Gandhi S, Poreddi V, Nikhil RS, Palaniappan M, Math SB. Indian novice nurses' perceptions of their role in caring for women who have experienced intimate partner violence. $\mathrm{Br} J$ Nurs. 2018;27(10):559-564. https://doi.org/10.12968/bjon. 2018.27.10.559. 
47. Blumling A, Kameg K, Cline T, Szpak J, Koller C. Evaluation of a standardized patient simulation on undergraduate nursing students' knowledge and confidence pertaining to intimate partner violence. J Forensic Nurs. 2018;14(3):174-179. https://doi.org/10.1097/JFN.0000000000000212.

48. Ryu JH, Lee JY, Song AY, Lee GY, Lee MJ. An integrated approach to abuse and violence across the lifespan. Sejong: Korea Institute for Health and Social Affaris; 2016.

49. Shin H, Lee JM, Kang KA, Kim SJ. An integrative literature review on sexual abuse prevention education programs for elementary school students in South Korea. Child Health Nurs Res. 2019;25(4):435-448. https://doi.org/10.4094/chnr. 2019.25.4.435.

50. Shin H, Lee JM, Min HY. An integrative literature review on sex education programs for Korean college students. J Korean Acad Soc Nurs Educ. 2020;26(1):78-96. https://doi.org/10. 5977/jkasne.2020.26.1.78. 